\title{
Respiratory Failure: A Rare Complication of Chronic Kidney Disease Mineral and Bone Disorder
}

\author{
Julian Yaxley, MBBS, ${ }^{1}$ Tahira Scott, MBBS $^{2}$ \\ ${ }^{1}$ Department of Nephrology, Gold Coast University Hospital, Gold Coast, Queensland, Australia ${ }^{2}$ Department of Nephrology, Townsville \\ Hospital, Townsville, Queensland, Australia
}

\begin{abstract}
Background: Disordered metabolism of bone and minerals is a problem frequently encountered in patients with chronic kidney disease. Early biochemical changes include altered calcium and phosphate balance, while advanced disease produces reduced bone strength and extraskeletal calcification. The syndrome describing this constellation of findings is termed chronic kidney disease mineral and bone disorder.

Case Report: This report details a rare and extreme manifestation of chronic kidney disease mineral and bone disorder in a patient on long-term hemodialysis for end-stage renal failure. Progressive abnormalities of the thoracic skeleton were ultimately severe enough to produce restrictive lung physiology and symptomatic respiratory failure.

Conclusion: Cases of chronic kidney disease mineral and bone disorder with pronounced clinical sequelae occur uncommonly in contemporary practice because of early detection and effective therapies. To our knowledge, this report is the first case in the literature of severe thoracic involvement manifesting as respiratory failure.
\end{abstract}

Keywords: Bone remodeling, hyperparathyroidism, kidney failure-chronic, osteodystrophy, respiratory insufficiency

Address correspondence to Julian Yaxley, MBBS, Department of Nephrology, Gold Coast University Hospital, 6 Callistris Court, Elanora, Queensland, 4221, Australia. Tel: +61 42-080-8049. Email: julianyaxley@yahoo.com.au

\section{INTRODUCTION}

Disordered bone and mineral metabolism is common in patients with chronic kidney disease (CKD). ${ }^{1}$ The spectrum of chemical and bony changes in these patients is referred to as CKD mineral and bone disorder (CKD-MBD). The pathophysiology is complex and comprises states of both high and low bone turnover. Derangements in serum calcium, phosphate, vitamin $D$, and parathyroid hormone $(\mathrm{PTH})$ are characteristic. Signs and symptoms in CKD-MBD generally occur only late in the disease course, and significant morbidity is uncommon in developed nations because of ready access to effective treatment.

We present the case of a patient on hemodialysis with uncontrolled CKD-MBD that was eventually complicated by a range of clinical sequelae rarely documented in the literature.

\section{CASE REPORT}

A 56-year-old indigenous Australian male with end-stage renal failure presented to the emergency department of a metropolitan Australian academic hospital complaining of lethargy for several weeks. He described progressive generalized weakness associated with worsening exertional dyspnea and chest tightness for the prior 2 months and an inability to manage the activities of daily living.

The patient had been on intermittent hemodialysis for 18 years after first presenting acutely in the year 2000 with renal failure and pulmonary edema requiring urgent dialysis. He remained dialysis-dependent from that point forward and never underwent kidney biopsy. Subtotal parathyroidectomy performed in 2008 for tertiary hyperparathyroidism was complicated by laryngeal nerve injury and vocal cord palsy, manifesting as voice hoarseness. Repeat parathyroidectomy was offered in $\mathbf{2 0 1 0}$ for recurrent hyperparathyroidism; however, the patient declined further surgical interventions.

He had a known provisional diagnosis of CKD-MBD, having previously had multiple vertebral insufficiency fractures and bilateral atraumatic femoral neck fractures for which he had undergone simultaneous hip hemiarthroplasty in 2016. Current medications were calcium carbonate $500 \mathrm{mg} 3$ times daily, calcitriol $0.25 \mathrm{mcg}$ daily, alendronate $70 \mathrm{mg}$ fortnightly, amlodipine $10 \mathrm{mg}$ daily, and subcutaneous darbepoetin alfa $80 \mathrm{mcg}$ fortnightly. He was prescribed cinacalcet $30 \mathrm{mg}$ twice daily from 2016-2017 until that medication was restricted by the Australian pharmaceutical benefits scheme. The patient was adherent with medications and dialysis requirements.

On examination, the patient had generalized muscle wasting with shallow respirations, but work of breathing was comfortable with no use of accessory muscles. Peripheral oxygen saturation on room air was $86 \%$, and his respiratory rate was $20 \mathrm{bpm}$. The patient had bony irregularities of multiple fingers, widened interdental spacing, and jaw enlargement. He had a marked thoracic kyphosis (Figure 1) and a 


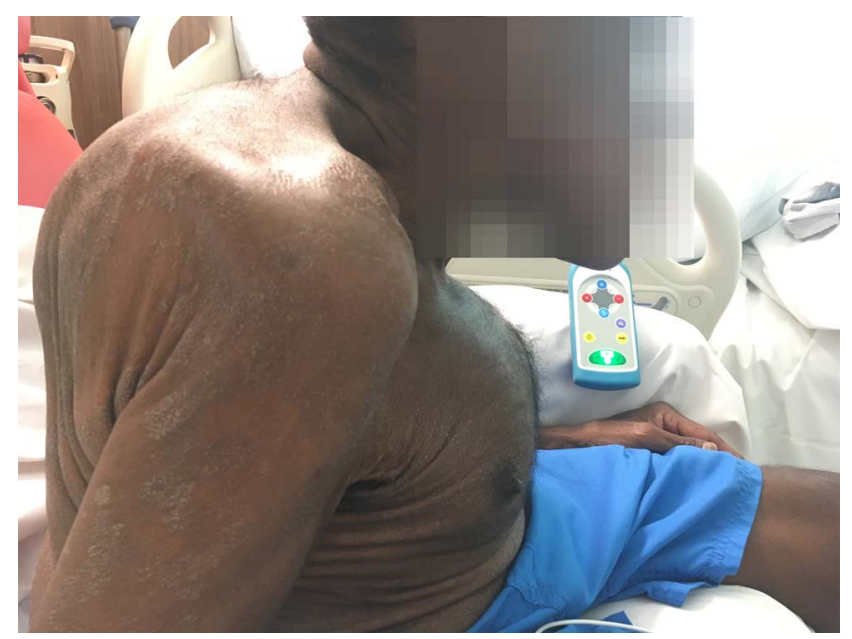

Figure 1. Pronounced vertebral kyphosis with macrognathia and chest wall deformities.

protuberant contorted chest wall contour (Figure 2). Chest expansion could not be appreciated on palpation, and breath sounds were reduced in the lower zones bilaterally. Heart sounds were normal. Muscle power was slightly reduced globally, and the patient developed increased dyspnea at a walking distance of only several meters.

His metabolic parameters were largely stable (Figure 3). Most recent corrected calcium was $2.52 \mathrm{mmol} / \mathrm{L}$ (normal range, 2.1-2.6 mmol/L), phosphate was $1.63 \mathrm{mmol} / \mathrm{L}$ (normal range, $0.75-1.5 \mathrm{mmol} / \mathrm{L}$ ), total alkaline phosphatase (ALP) was 1,070 U/L (normal range, 30-110 U/L), 25-hydroxyvitamin D was $64 \mathrm{nmol} / \mathrm{L}$ (normal range, $50-150 \mathrm{nmol} / \mathrm{L}$ ), and $\mathrm{PTH}$ was $480 \mathrm{pmol} / \mathrm{L}$ (normal range, 1.0-7.0 pmol/L). Bone-specific ALP and fibroblast growth factor 23 were not measured. Arterial blood gas sampling confirmed chronic respiratory acidosis with hypercapnic respiratory failure. Computed tomography (CT) pulmonary angiogram showed pronounced scoliosis and kyphosis, multilevel endplate vertebral sclerosis, chest wall distortion with low density dysplastic rib architecture, and patchy subcutaneous cal-

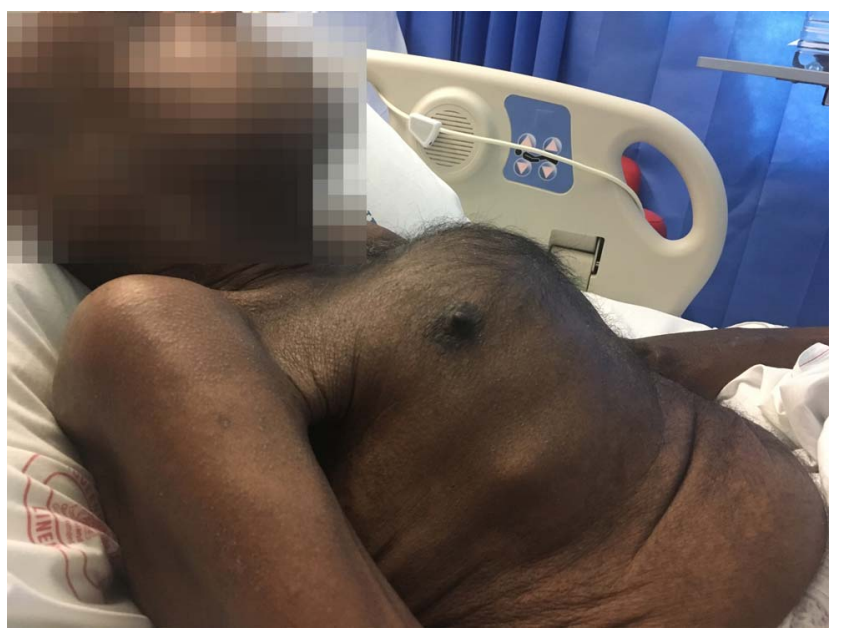

Figure 2. Petcus carinatum with expansion of the manubrium and sternum.

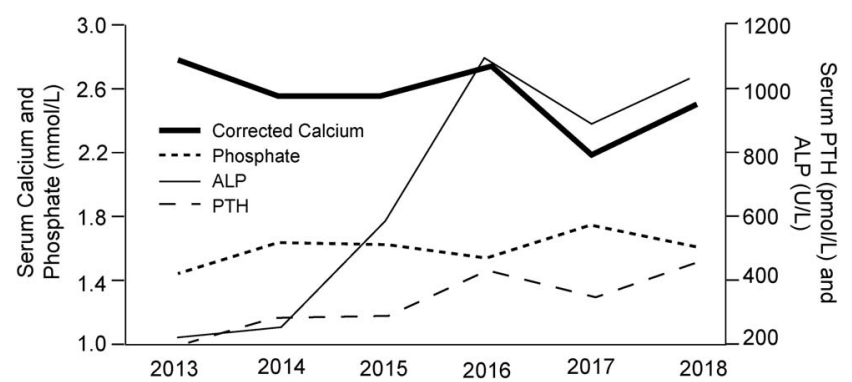

Figure 3. Serum metabolic parameters over time; note the improvement in parathyroid hormone (PTH) and alkaline phosphatase (ALP) from 2016-2017 during treatment with cinacalcet.

cification. The patient had bibasilar atelectasis and significant coronary and aortic calcification but no evidence of pulmonary edema, pulmonary hypertension, embolism, or other parenchymal abnormalities (Figure 4). There were no bone cysts to suggest dialysis-related amyloidosis or brown tumors. Skeletal deformities were less prominent on a CT performed a year prior. Transthoracic echocardiography showed normal biventricular function with aortic sclerosis and mitral annular calcification. Spirometry was performed with difficulty. The patient recorded a mean forced expiratory volume in one second $\left(\mathrm{FEV}_{1}\right)$ of $0.40 \mathrm{~L}(16 \%$ of expected reference of $2.50 \mathrm{~L}$ ) and mean forced vital capacity (FVC) of $0.61 \mathrm{~L}$ ( $18 \%$ of expected reference of $3.38 \mathrm{~L})$, for an $\mathrm{FEV}_{1}: \mathrm{FVC}$ ratio of $73 \%$. Despite the patient's best attempts, his effort was too inadequate to measure diffusing capacity.

Severe restrictive lung disease was diagnosed, thought to be secondary to a restrictive ventilatory defect caused by malformation of the thoracic skeleton and soft tissue ossification, ostensibly as a result of advanced CKD-MBD. Because of deconditioning, the patient received physiotherapy and rehabilitation for 10 weeks prior to discharge. He was prescribed domiciliary oxygen, and despite previously living independently, the patient required nursing home placement because of frailty.

\section{DISCUSSION}

CKD-MBD is a systemic perturbation in bone and mineral handling in patients with renal impairment. The pathobiology of CKD-MBD is complex, and knowledge is continually evolving. The spectrum of lesions within CKD-MBD range from states of high bone turnover, principally osteitis fibrosa and tertiary hyperparathyroidism, to states of low bone turnover, such as adynamic bone disease, extraskeletal calcification, osteoporosis, and osteomalacia. The pathologic state is mixed in most cases. Mid-stage CKD with secondary hyperparathyroidism and osteitis fibrosa typically causes low serum calcium with phosphate retention, while advanced CKD with tertiary hyperparathyroidism can cause high calcium and normalization of phosphate. Adynamic bone disease appears similar, but PTH is relatively suppressed. The structural repercussion is low bone strength and abnormal volume. Renal osteodystrophy refers specifically to bone histomorphologic features in a patient with the clinical syndrome of CKD-MBD, with each of the various bone turnover states characterized by specific findings. 

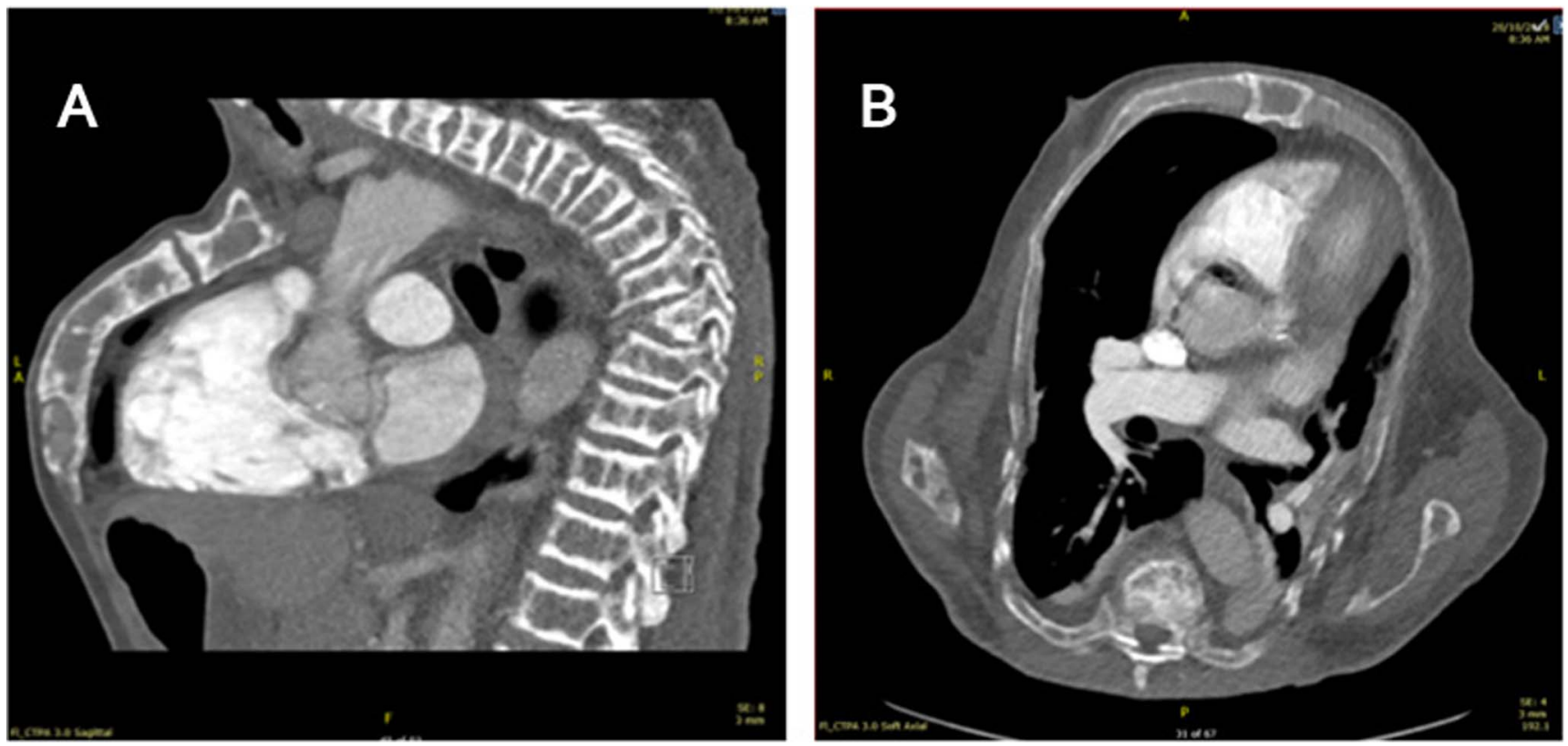

Figure 4. Chest computed tomography demonstrates $(A)$ thoracic compression fracture and (B) irregular thoracic bone architecture.

The prevalence of CKD-MBD increases with declining renal function. Most subjects with a glomerular filtration rate $\left(\right.$ GFR) $<60 \mathrm{~mL} / \mathrm{min} / 1.73 \mathrm{~m}^{2}$ have some evidence of the disorder, and bone disease is almost universal in patients with end-stage renal failure. ${ }^{1}$ CKD-MBD is generally asymptomatic for many years ${ }^{2}$ and probably is associated with lower morbidity in westernized health systems such as those of Australia or the United States with ready access to surveillance and treatment. ${ }^{3}$ Signs and symptoms, if present, may include myopathy; bone pain and joint stiffness; visible bone remodeling; fractures; and extraskeletal calcification in the form of deposition in skin, soft tissues, or vasculature.

The gold standard means of confirming diagnosis is bone biopsy, although this procedure is rarely done in daily practice because of availability and cost constraints. Diagnosis is more commonly made using an accumulation of other indirect evidence. Blood tests that offer some guidance include PTH, ALP, bone-specific ALP, and dynamics in serum calcium and phosphate levels. An increasing PTH level implies the development of secondary or tertiary hyperparathyroidism, and a subsequent decrease could imply iatrogenic oversuppression and adynamic bone. In patients with CKD, elevated ALP implies an abnormal state of excess osteoblast activity, although bone-specific ALP is a more accurate measure because it is not influenced by liver disease. Radiologic findings indicating renal osteodystrophy include subperiosteal resorption, sclerosis, and osteopenia. ${ }^{4}$

Management of CKD-MBD involves long-term dietary moderation of calcium and phosphate consumption. Various medications are also used to achieve a balance in serum calcium, phosphate, and PTH. These medications include phosphate binders, vitamin D metabolites, and calcimimetics to inhibit PTH secretion. Parathyroidectomy is required for patients with tertiary hyperparathyroidism refractory to conservative therapy, and a significant minority of patients require reoperation because of recurrence.

The patient in this case report illustrates classic features of advanced disease, now rarely seen in the Australian setting. In the majority of patients, severe morbidity can be averted, and treatment refractoriness is rare. Our patient progressed despite optimal medical management, albeit remaining undertreated because of his preference to avoid repeat parathyroid surgery. His was primarily a state of high bone turnover, as evidenced by elevated parahormone, but his long-term serum calcium and phosphate balance remained relatively stable on phosphate binders and dialysis. On physical examination, the patient had reduced strength consistent with myopathy, macrognathia, and bony finger changes. The patient also had an important history of atraumatic femoral neck fractures. He had radiographic evidence of vascular calcification but no clinical complications which is unusual given that the majority of dialysis patients with radiologically proven vessel calcification experience cardiovascular events within 12 years. ${ }^{5}$ Interestingly, although the patient experienced dramatic improvement in PTH during the 12 months that he was prescribed cinacalcet, his bony deformities proceeded nonetheless.

Respiratory failure is a complication of CKD-MBD not previously described in the medical literature. On the other hand, subclinical axial involvement is widely appreciated in patients with renal osteodystrophy. More than $60 \%$ of patients on dialysis have extraskeletal thoracic calcification at autopsy. ${ }^{6}$ A 2018 epidemiologic study found that $36 \%$ of patients with a GFR $<15 \mathrm{~mL} / \mathrm{min} / 1.73 \mathrm{~m}^{2}$ demonstrate asymptomatic restrictive indices on spirometry, presumably attributable to the various processes outlined above. ${ }^{7}$ Importantly, most patients did not have coexisting primary lung disease. Symptomatic respiratory failure is unique and 
represents a severe manifestation of CKD-MBD. Several mechanisms are likely to be implicated in the reported case. In this patient, extraskeletal calcification, ribcage defects, multiple vertebral insufficiency fractures, and a prominent thoracic kyphosis were significant enough to cause restrictive physiology and atelectasis. Other postulated mechanisms potentially contributing to this patient's symptoms include subclinical pulmonary edema, diaphragmatic palsy, and respiratory muscle wasting due to uremia.,

\section{CONCLUSION}

CKD-MBD is a systemic disorder in the metabolism of bone and minerals that is common in patients with CKD. Most aspects of its evaluation and management relate to controlling biochemical changes, as CKD-MBD is generally asymptomatic for many years. Advanced clinical sequelae are rare in contemporary practice; however, some cases may remain refractory to all treatment options. This case is unique to the literature. It details a patient with advanced thoracic involvement producing restrictive lung physiology and symptomatic chronic respiratory failure. This case serves as a reminder to consider the substantial morbidity that may accompany CKD-MBD in all patients with CKD.

\section{ACKNOWLEDGMENTS}

The authors have no financial or proprietary interest in the subject matter of this article.

\section{REFERENCES}

1. Isakova T, Wahl P, Vargas GS, et al. Fibroblast growth factor 23 is elevated before parathyroid hormone and phosphate in chronic kidney disease. Kidney Int. 2011 Jun;79(12):1370-1378. doi: 10.1038/ki.2011.47.

2. Martin KJ, Gonzalez EA. Metabolic bone disease in chronic kidney disease. J Am Soc Nephrol. 2007 Mar;18(3):875-885. doi: 10.1681/ASN.2006070771.

3. Kher V. End-stage renal disease in developing countries. Kidney Int. 2002 Jul;62(1):350-362. doi: 10.1046/j.1523-1755.2002.00426.x.

4. Murphey MD, Sartoris DJ, Quale JL, Pathria MN, Martin NL. Musculoskeletal manifestations of chronic renal insufficiency. Radiographics. 1993 Mar;13(2):357-379. doi: 10.1148/radiographics.13.2.8460225.

5. Schwaiger JP, Neyer U, Sprenger-Mahr H, et al. A simple score predicts future cardiovascular events in an inception cohort of dialysis patients. Kidney Int. 2006 Aug;70(3):543-548. doi: 10.1038/sj.ki.5001589.

6. Gavelli G, Zompatori M. Thoracic complications in uraemic patients and in patients undergoing dialytic treatment: state of the art. Eur Radiol. 1997;7(5):708-717. doi: 10.1007/BF02742931.

7. Mukai $H$, Ming $P$, Lindholm B, et al. Restrictive lung disorder is common in patients with kidney failure and associates with protein energy wasting, inflammation and cardiovascular disease. PLoS One. 2018 Apr 27;13(4):e0195585. doi: 10.1371/journal.pone.0195585.

This article meets the Accreditation Council for Graduate Medical Education and the American Board of Medical Specialties Maintenance of Certification competencies for Patient Care and Medical Knowledge. 